\title{
Using Diaper Waste to Prepare Magnetic Catalyst for the Synthesis of Glycerol Carbonate
}

\author{
Jincan Wang, ${ }^{1}$ Yong Liang, ${ }^{2}$ Song Wang $\left({ }^{1}{ }^{1}\right.$ Patrick U. Okoye, ${ }^{3}$ Hongxu Chen, ${ }^{1}$ Yue Zhou, ${ }^{1}$ \\ Jiening $\mathrm{Xu}^{1}{ }^{1}$ Zhenhao Meng, ${ }^{1}$ Lei Wang, ${ }^{1}$ and Sanxi $\mathrm{Li}^{1}$ \\ ${ }^{1}$ School of Environmental and Chemical Engineering, Shenyang University of Technology, Shenyang 110870, China \\ ${ }^{2}$ Liaoning Hanking Green Building Materials Co., LTD., Fushun 113000, China \\ ${ }^{3}$ Laboratorio de Bioenergía, Instituto de Energías Renovables (IER-UNAM), Temixco, Morelos 62580, Mexico
}

Correspondence should be addressed to Song Wang; 1259669134@qq.com

Received 9 July 2020; Revised 18 September 2020; Accepted 17 October 2020; Published 30 October 2020

Academic Editor: Ulrich Maschke

Copyright (c) 2020 Jincan Wang et al. This is an open access article distributed under the Creative Commons Attribution License, which permits unrestricted use, distribution, and reproduction in any medium, provided the original work is properly cited.

\begin{abstract}
Diaper waste was calcined above $400^{\circ} \mathrm{C}$ after impregnated in the solution of nickel nitrate. The as-prepared diaper waste-derived materials were used as magnetic catalysts for the synthesis of glycerol carbonate (GC). Structure and catalytic ability investigations on the catalysts calcined at different temperatures indicated that calcination temperature was an important factor affecting the property of catalysts. It was found that the catalyst obtained at the calcination temperature of $700^{\circ} \mathrm{C}$ (named $\left.\mathrm{DW}-\mathrm{Ni}-700\right)$ showed the best performance. When DW-Ni-700 was used in the synthesis of GC, GC yield reached 93.2\%, and the magnetic property of DW-Ni-700 facilitated the catalyst separation process. Meanwhile, DW-Ni-700 showed high reusability in the reaction. After four times reuse of DW-Ni-700, GC yield decreased less than $4 \%$.
\end{abstract}

\section{Introduction}

In the past few decades, to meet the increasing demand for the eco-friendly energy, biodiesel industry was developed rapidly $[1,2]$. With the development of biodiesel industry, some problems arose. One of these problems is that how to deal with the main by-product of the biodiesel industry, glycerol $[3,4]$. In some countries, glycerol was dumped directly, leading to the serious environmental problems $[5,6]$. Therefore, how to efficiently use by-product, glycerol, has become an important problem affecting the development of biodiesel industry $[7,8]$.

Using glycerol to produce value-added chemical products is a solution. Glycerol carbonate (GC) is such a chemical product, which can be produced from glycerol. Several methods have been reported to produce GC from glycerol [9]. Among these methods, due to the high GC yield and mild reaction condition, using the transesterification of glycerol with dimethyl carbonate (DMC) to produce GC has drawn more and more attention [10]. During the investigation on the transesterification of glycerol with DMC, it was found that base catalyst can greatly affect the produced GC quality. Homogeneous catalysts such as $\mathrm{KOH}$ and $\mathrm{NaOH}$ can greatly accelerate the reaction, leading to the high GC yield. However, it is difficult to separate these homogeneous catalysts with GC product.

Thus, heterogeneous catalysts, including alkali metal oxides, hydrotalcites, ionic liquids, alkali metal salts, and lipase, were developed as the alternative of homogeneous catalysts $[11,12]$. In recent years, waste-derived materials have attracted more and more attention because of the negligible cost of feedstock [13] Some industrial waste, agricultural waste, and municipal waste have been used as the feedstock to prepare catalyst, which showed similar catalytic performance as the catalysts prepared from the pure chemicals $[14,15]$. Compared with homogeneous catalysts, all these developed heterogeneous catalysts have the advantages of easy separation and low pollution. Nevertheless, to reuse these heterogeneous catalysts, centrifugation or filtration methods are needed, which not only consumes energy and time but also causes the loss of catalyst [16]. 
To solve this problem, magnetic heterogeneous catalyst (MHC) was developed, which can be efficiently separated from the reaction system with less catalyst loss by applying an external magnetic field [15, 17-19]. Rezayan et al. prepared a zeolite-supported $\mathrm{MHC}, \mathrm{KOH} / \mathrm{ZSM} 5-\mathrm{Fe}_{3} \mathrm{O}_{4}$, which showed high catalytic ability and separation efficiency in the synthesis of biodiesel [20]. Tudorache et al. immobilized lipase onto the surface of magnetic particle to prepare a bioMHC and successfully used it to synthesize GC [21]. Although MHCs exhibited obvious advantages in the catalyst separation process, the cost and complex preparation procedure led the large-scale application of MHC difficult. Therefore, it is a requirement to find a simple and low-cost method to prepare MHC.

Diaper waste is one of the typical municipal wastes. In some countries, diaper waste accounts for about $6 \%$ of municipal waste [22]. As a product prepared from polymer materials, diaper is mainly composed of fluff pulp, super absorbent resin (sodium polyacrylate), and non-woven polypropylene, which are non-biodegradable. Direct disposal of diaper waste can cause environmental problems [23]. Therefore, many investigations on how to utilize diaper waste scientifically were carried out. By now, it has been found that diaper waste can be used as the feedstock of compost and anode material $[24,25]$. Finding new methods to utilize diaper waste is attracting more and more attention.

Recently, using polymer material to prepare catalyst has drawn more and more attention [26]. In this work, a polymeric waste, diaper waste, was used as feedstock to prepare $\mathrm{MHC}$ for the first time. After being impregnated in the solution of nickel nitrate, diaper waste was calcined above $400^{\circ} \mathrm{C}$ to prepare a series of base MHCs. X-ray diffraction (XRD), the Fourier-transform infrared spectra (FTIR), the BrunauerEmmet-Teller specific surface area analyze (BET), scanning electron microscope (SEM), energy-dispersive X-ray spectrum (EDX), magnetic performance test, and acid-base titration technique were applied to characterize the structure of the MHCs. Moreover, the magnetic separation and catalytic ability of the MHCs were compared. The MHC, which exhibited the best performance, was selected to investigate the effect of the reaction condition on the synthesis of GC and the reusability of the diaper waste-derived $\mathrm{MHC}$ in the reaction.

\section{Materials and Methods}

2.1. Materials. All the reagents, including DMC, glycerol, nickel nitrate, and methanol, were purchased from Tianjin Damao Chemical Reagent Co., China. Diaper waste used in this work was acquired from the local municipal waste.

2.2. Catalyst Preparation. To prepare the MHC, diaper waste was firstly dried at $100^{\circ} \mathrm{C}$ for $4 \mathrm{~h}$ to remove the adsorbed water, and the dried diaper waste was cut into small pieces. Then, diaper waste was impregnated into the nickel nitrate solution of $0.5 \mathrm{~mol} / \mathrm{L}$ with the ratio of $5 \mathrm{~g}$ diaper waste to $10 \mathrm{mmol}$ nickel nitrate. After being impregnated at room temperature for $12 \mathrm{~h}$, the fully impregnated diaper waste was dried at $110^{\circ} \mathrm{C}$ for $6 \mathrm{~h}$. The diaper waste doped with nickel nitrate was calcined above $400^{\circ} \mathrm{C}$ in a nitrogen atmo- sphere to prepare the MHCs. The as-prepared MHC was named DW-Ni- $t$, where $t$ indicated the calcinations temperature. For example, the catalyst obtained at the calcination temperature of $700^{\circ} \mathrm{C}$ was named DW-Ni-700.

2.3. Characterization of Catalyst. Scanning electron microscope (Phenom Pro X, Netherlands) was used to observe the surface morphology of the catalyst calcined at different temperatures. The element composition was detected by energy-dispersive X-ray spectrum fitted with the same equipment. The functional group of catalyst was investigated by an infrared analyzer (IR-prestige21, Japan). The KBr tabletting method was used to prepare sample and the scanning scope was $4000-400 \mathrm{~cm}^{-1}$. To investigate the crystal structure of catalyst, the X-ray diffraction instrument (X. Pert Pro Mpo PW3040, Netherlands) was used at $40 \mathrm{kV}$ and $30 \mathrm{~mA}$. All MHCs were scanned in the $2 \theta$ range of $5^{\circ}-80^{\circ}$ at a scanning speed of $4^{\circ} \mathrm{min}^{-1}$. A vibrating sample magnetometer (Lake Shore 7410, USA) was used to characterize the magnetic property of MHCs. Specific surface area of catalyst was measured by a nitrogen adsorption/desorption isotherms at $77 \mathrm{~K}$ by the Brunauer-Emmet-Teller method (JW-BK122W, China). Before analysis, the catalysts were degassed at $120^{\circ} \mathrm{C}$ for $2 \mathrm{~h}$ to remove moisture absorbed on the surface of samples. The acid-base titration technique was carried out according to the reported literature [27].

2.4. Transesterification of Glycerol with DMC. Transesterification reaction of glycerol with DMC was carried out in a $100 \mathrm{~mL}$ three-necked flask, which was fitted with a thermometer, reflux condenser, and mechanical stirrer. To synthesis GC, the mixture of glycerol $(9.2 \mathrm{~g}, 0.1 \mathrm{~mol}), \mathrm{DMC}(27.0 \mathrm{~g}$, $0.3 \mathrm{~mol})$, and the MHC (1.1 g, $3 \mathrm{wt} . \%)$ was thermal heated at $80^{\circ} \mathrm{C}$ and stirred at $500 \mathrm{rpm}$ for $60 \mathrm{~min}$. After that, the reaction was stopped, and the MHC was magnetic separated from the product using a magnet. A gas chromatography (Agilent GC-7890A, USA) was applied to determine the composition of the product. GC yield and glycerol conversion were calculated as reported in literatures [28].

\section{Results and Discussion}

3.1. Characterization of Catalysts. The surface morphology of diaper waste-derived MHCs is presented in Figure 1. According to the SEM images, the surface of DW-Ni-400 was relatively smooth, and no obvious pore structure can be observed on its surface, which indicated the incomplete decomposition of feedstock. When the calcination temperature was higher than $400^{\circ} \mathrm{C}$, more and more pore structures can be observed on the surface of catalyst. The foamy-like morphology can be observed on the surface of DW-Ni-700 and DW-Ni-800. Moreover, the EDX mapping images indicated that the $\mathrm{Ni}$ and $\mathrm{Na}$ elements dispersed uniformly on MHCs. The SEM results demonstrated that when the calcination temperature was higher than $400^{\circ} \mathrm{C}$, the decomposition of the feedstock became violent, leading to the disappearance of the smooth surface morphology.

The FTIR spectra of the catalysts is shown in Figure 2. All of these catalysts exhibited similar FTIR pattern; the band at 


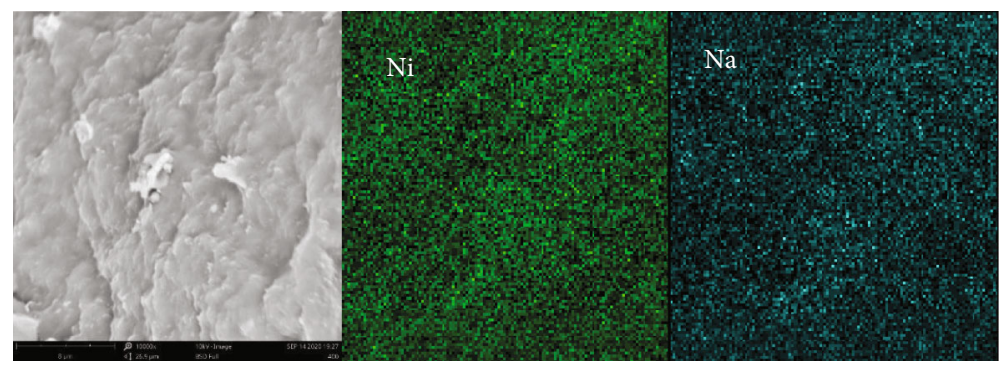

(a)

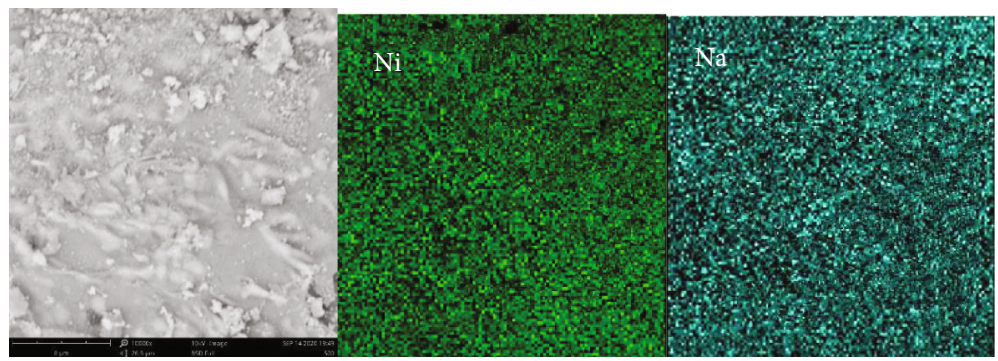

(b)

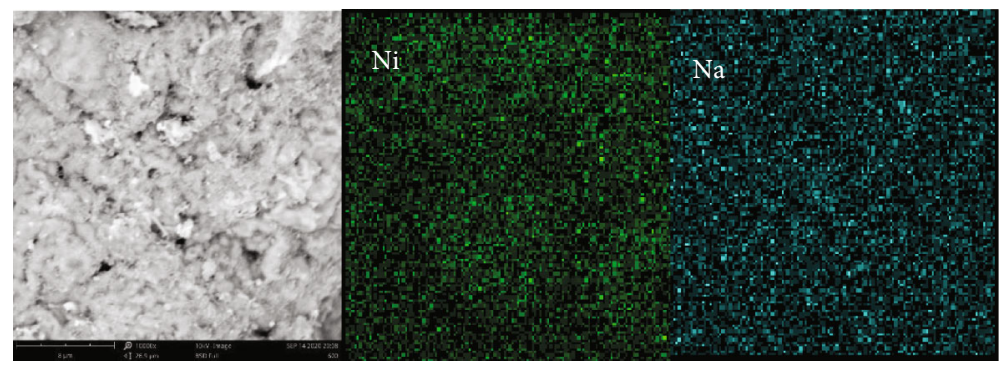

(c)

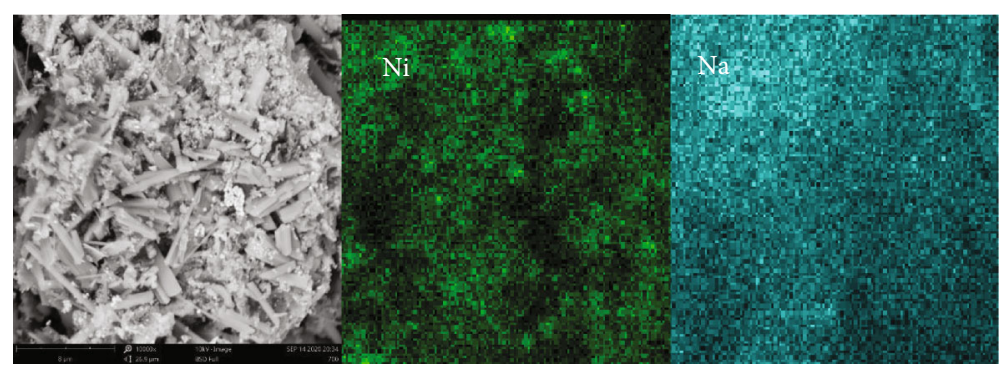

(d)

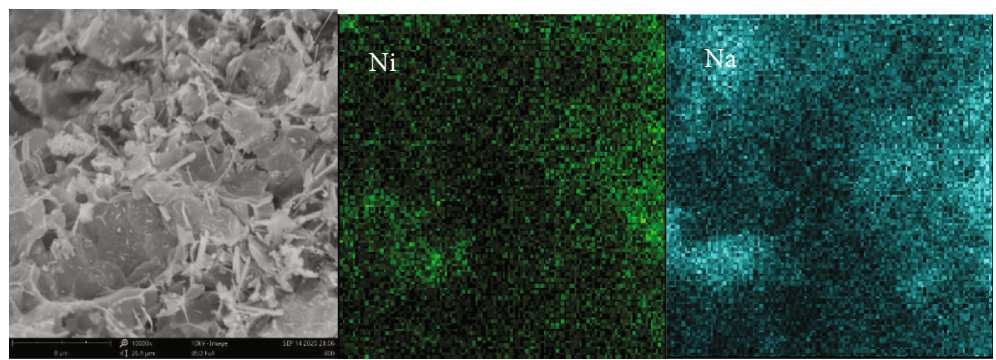

(e)

FIGURE 1: SEM images and corresponding EDX elemental mapping of (a) DW-Ni-400, (b) DW-Ni-500, (c) DW-Ni-600, (d) DW-Ni-700, and (e) DW-Ni-800.

$3433 \mathrm{~cm}^{-1}$ was attributed to the vibration of hydroxyl bond. The bands at $1620 \mathrm{~cm}^{-1}, 1437 \mathrm{~cm}^{-1}$, and $880 \mathrm{~cm}^{-1}$ were assigned to the vibration of carbonate $[17,29]$. The FTIR spectrum of DW-Ni-400 was a little different from other catalysts. When DW-Ni-400 was examined, there were two extra bands that can be observed. One band was at 


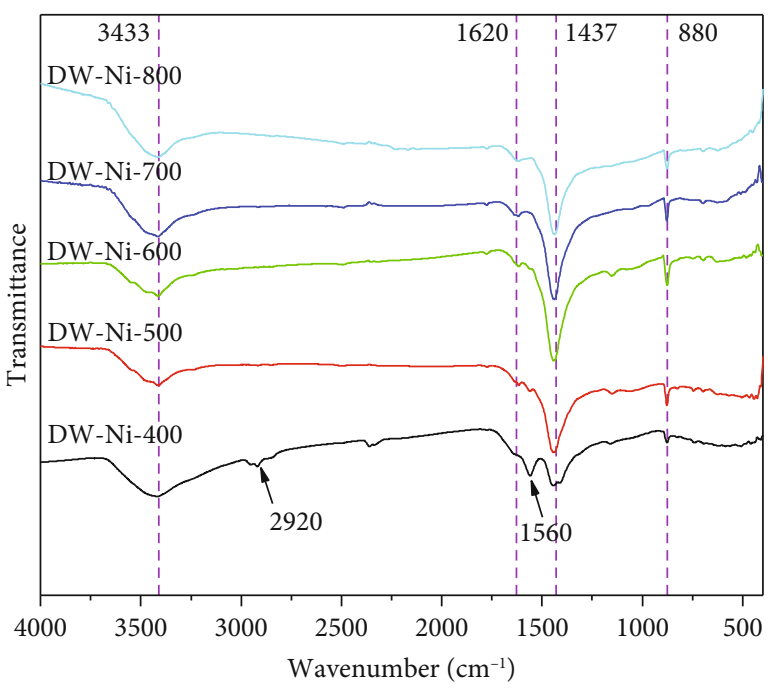

Figure 2: FTIR spectra of the catalysts prepared at different calcination temperatures.

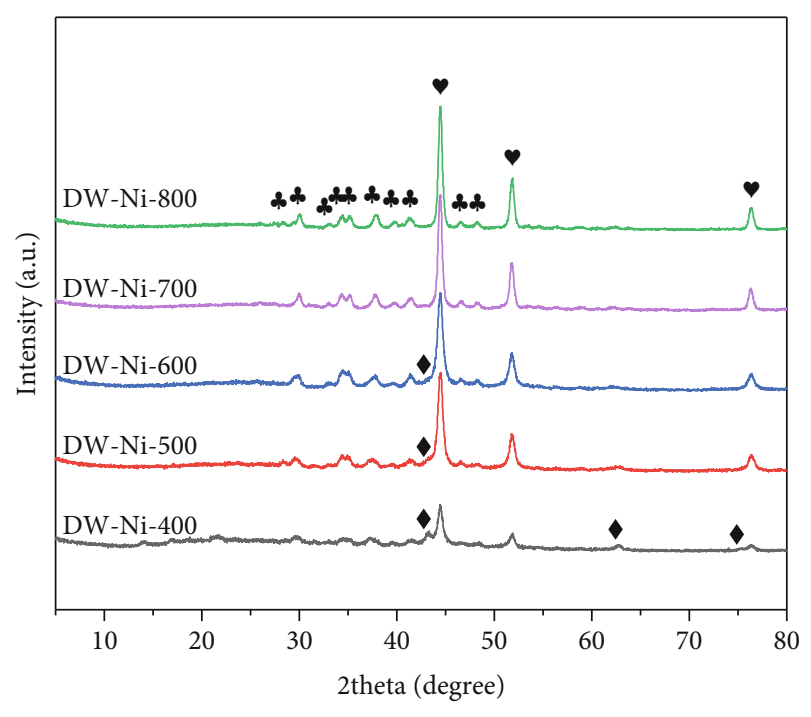

- $\mathrm{Ni}$

+ $\mathrm{Na}_{2} \mathrm{CO}_{3}$

$\checkmark \mathrm{NiO}$

FIGURE 3: XRD of the catalysts prepared at different calcination temperatures.

$2920 \mathrm{~cm}^{-1}$, and another band was at $1560 \mathrm{~cm}^{-1}$. Since the bands at $2920 \mathrm{~cm}^{-1}$ and $1560 \mathrm{~cm}^{-1}$ can be attributed to the vibration of $\mathrm{C}-\mathrm{H}$ and $\mathrm{N}-\mathrm{H}$ bonds $[30,31]$, respectively, the existence of these two bands indicated that a certain amount of undecomposed organic compound was contained in DW-Ni-400. The FTIR investigation results indicated that organic compound in diaper waste can be totally decomposed above $500^{\circ} \mathrm{C}$, and carbonate was formed during the calcinations process.

Figure 3 shows the XRD spectra of the catalysts calcined at different temperatures. In the diffraction pattern of DW$\mathrm{Ni}-400$, the diffraction peaks that appeared at $2 \theta$ of $43.3^{\circ}$, $62.5^{\circ}$, and $75.5^{\circ}$ were assigned to the diffraction of $\mathrm{NiO}$ (JCPDS 75-0197). The sharp diffraction peaks that appeared

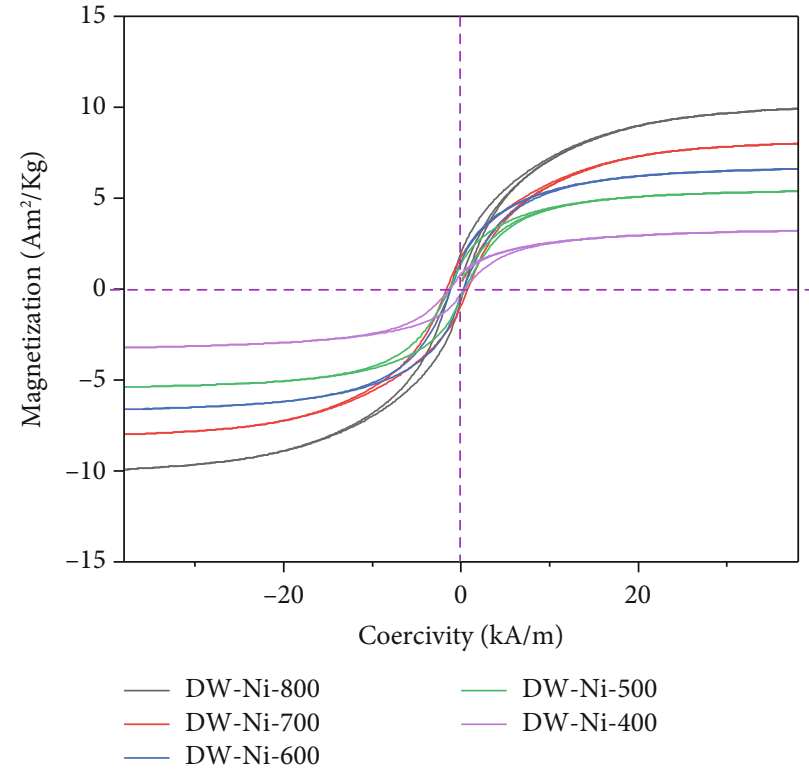

FIgURE 4: Hysteresis loop of the catalysts prepared at different calcination temperatures.

TABle 1: Physicochemical properties of catalysts prepared at different calcination temperatures.

\begin{tabular}{lccc}
\hline Catalyst & $\begin{array}{c}\mathrm{Ms} \\
\left(\mathrm{Am}^{2} / \mathrm{kg}\right)\end{array}$ & $\begin{array}{c}\text { BET surface area } \\
\left(\mathrm{m}^{2} / \mathrm{g}\right)\end{array}$ & $\begin{array}{c}\text { Total basicity } \\
(\mathrm{mmol} / \mathrm{g})\end{array}$ \\
\hline DW-Ni-800 & 9.8 & 26.7 & 4.8 \\
DW-Ni-700 & 7.9 & 59.9 & 4.7 \\
DW-Ni-600 & 6.6 & 56.6 & 4.6 \\
DW-Ni-500 & 5.3 & 27.2 & 3.4 \\
DW-Ni-400 & 3.2 & 8.4 & 2.1 \\
\hline
\end{tabular}

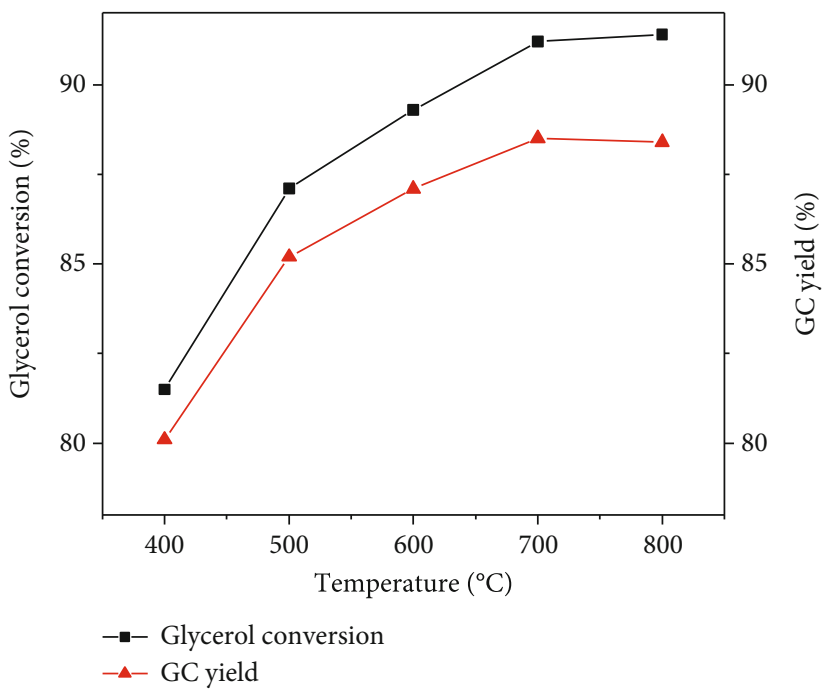

FIGURE 5: The catalytic activity of the DW-Ni catalysts. Reaction conditions: glycerol: $0.1 \mathrm{~mol}$ (9.2 g), DMC: $0.3 \mathrm{~mol}(27.0 \mathrm{~g})$, catalyst: 3 wt. $\%$ (1.08 g), temperature: $75^{\circ} \mathrm{C}$, and time: $1.5 \mathrm{~h}$. 


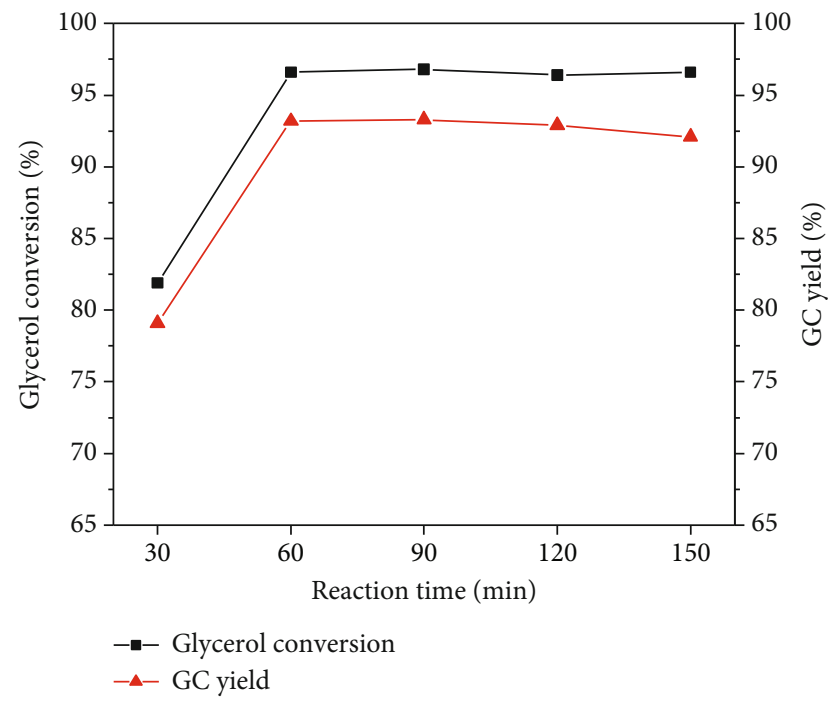

(a)

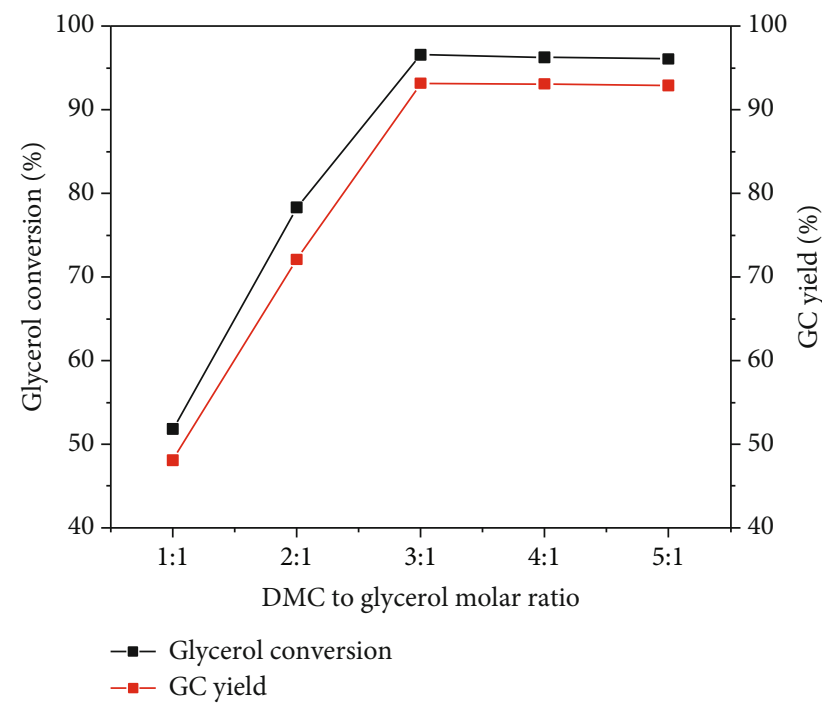

(c)

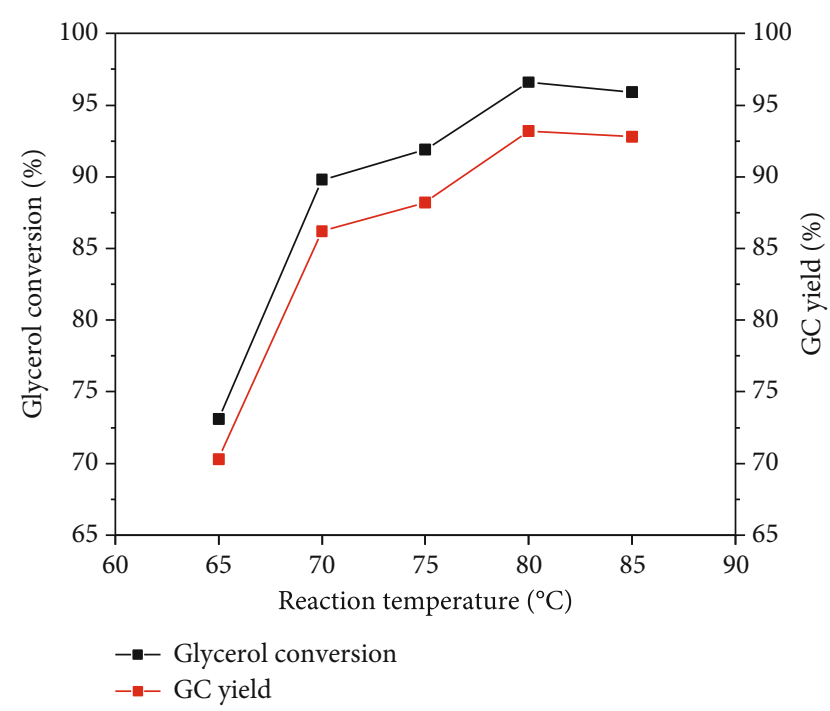

(b)

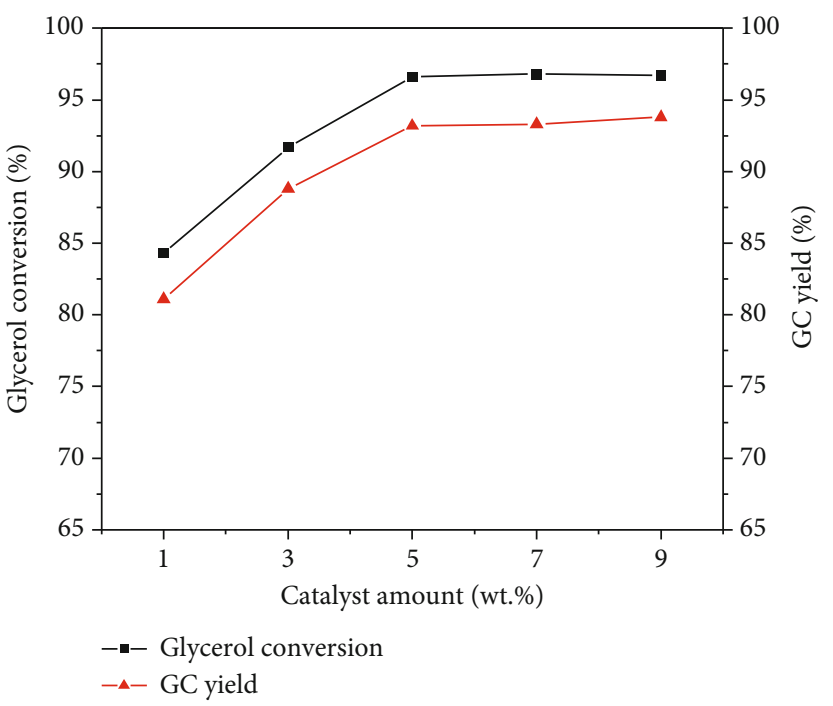

(d)

FIGURE 6: Effect of reaction condition on the synthesis of GC. (a) Effect of reaction time. (b) Effect of reaction temperature. (c) Effect of DMC to glycerol molar ratio. (d) Effect of Catalyst amount. The detailed reaction condition was listed in Table S1.

at $44.5^{\circ}, 51.4^{\circ}$, and $76.4^{\circ}$ indicated the existence of $\mathrm{Ni}$ (JCPDS87-0712) [32]. The weak diffraction peaks of $\mathrm{Na}_{2} \mathrm{CO}_{3}$ can be observed at $27.6^{\circ}, 30.1^{\circ}, 33.0^{\circ}, 34.5^{\circ}, 35.2^{\circ}, 37.8^{\circ}, 39.7^{\circ}$, $41.5^{\circ}, 48.3^{\circ}$, and $46.6^{\circ}$ (JCPDS 77-2082). Another phenomenon can be observed from Figure 3 was that, with the calcination temperature increased from $400^{\circ} \mathrm{C}$ to $800^{\circ} \mathrm{C}$, the strength of the diffraction peaks corresponding to $\mathrm{Ni}$ and $\mathrm{Na}_{2} \mathrm{CO}_{3}$ increased while the strength of the diffraction peaks corresponding to $\mathrm{NiO}$ decreased. When the calcination temperature was higher than $700^{\circ} \mathrm{C}$, no diffraction peaks of $\mathrm{NiO}$ can be observed. Moreover, the broad peak appeared at around $23^{\circ}$ (Figure S1) demonstrated the existence of carbon material, which was composed by amorphous carbon and graphitized carbon [33]. The existence of these diffraction peaks indicated that DW-Ni-400, DW-Ni-500, and DW-Ni-600 were mainly composed of $\mathrm{Ni}, \mathrm{NiO}$, $\mathrm{Na}_{2} \mathrm{CO}_{3}$, and carbon while DW-Ni-700 and DW-Ni-800 were mainly composed of $\mathrm{Ni}, \mathrm{Na}_{2} \mathrm{CO}_{3}$, and carbon (Scheme S1). In diaper waste-derived MHCs, $\mathrm{Na}_{2} \mathrm{CO}_{3}$ can provide the necessary basicity for the synthesis of GC, and the low solubility of $\mathrm{Na}_{2} \mathrm{CO}_{3}$ in methanol and glycerol made it a good heterogeneous catalyst [34]. The existence of Ni made MHCs magnetic, and amorphous carbon was the suitable supporter which can stabilize basic sites [16, 35]. The existence of $\mathrm{NiO}$ had no benefit for improving the catalyst performance. Thus, DW-Ni-700 and DW-Ni-800, which were the composite of $\mathrm{Ni}, \mathrm{Na}_{2} \mathrm{CO}_{3}$, and carbon, could show much better performance in the synthesis of GC.

Figure 4 presents the hysteresis loops of the catalysts obtained at different calcination temperatures. It can be seen from the diagram that the catalyst obtained at the higher calcination temperature exhibited the higher saturation magnetization (Ms). As shown in Table 1, the Ms of DW-Ni-800 and DW-Ni-700 was higher than $7 \mathrm{Am}^{2} / \mathrm{kg}$ while the Ms of 
DW-Ni-400, DW-Ni-500, and DW-Ni-600 was lower than $7 \mathrm{Am}^{2} / \mathrm{kg}$. Since the Ms of the catalyst was higher than $7 \mathrm{Am}^{2} / \mathrm{kg}$, the magnetic separation of catalyst can be performed easily [36]; the VSM investigation of the catalysts indicated that DW-Ni-800 and DW-Ni-700 could be excellent MHC.

The specific surface area and total basicity of the catalysts calcined at different temperatures were determined, and the results are shown in Table 1 . With the increase of calcination temperature from $400^{\circ} \mathrm{C}$ to $800^{\circ} \mathrm{C}$, the specific surface area of the corresponding catalyst firstly increased and then decreased. When the calcination temperature rose from $400^{\circ} \mathrm{C}$ to $700^{\circ} \mathrm{C}$, with the decomposition of feedstock, the specific surface area increased from $8.4 \mathrm{~m}^{2} / \mathrm{g}$ to $59.9 \mathrm{~m}^{2} / \mathrm{g}$. This was in accordance with the SEM results that pore structure was formed during calcination process. When the calcination temperature rose to $800^{\circ} \mathrm{C}$, the specific surface area decreased to $26.7 \mathrm{~m}^{2} / \mathrm{g}$. This was due to the excessive decomposition of carbon material formed during the calcination process, leading to the pore collapse and the decrease of specific surface area. To obtain high specific surface area catalyst, the calcination temperature should not be higher than $800^{\circ} \mathrm{C}$. Furthermore, it can be seen from Table 1 that the total basicity of the catalysts increased with the calcination temperature. The total basicity of DW-Ni-600, DW-Ni-700, and DW-Ni800 was all higher than $4.5 \mathrm{mmol} / \mathrm{g}$, while the total basicity of DW-Ni-400 and DW-Ni-500 was lower than $3.5 \mathrm{mmol} / \mathrm{g}$. The difference of the specific surface area and total basicity between the prepared catalysts can greatly affect their catalytic ability.

The catalytic performance of the diaper waste-derived MHCs in the synthesis of GC was examined and the corresponding results are presented in Figure 5. Both DW-Ni700 and DW-Ni-800 showed high catalytic performance with the GC yield of higher than 90\%, while the GC yield was lower than $90 \%$ when DW-Ni-400, DW-Ni-500, and DWNi-600 were used. Compared with DW-Ni-800, DW-Ni-700 can be obtained at the relatively low calcination temperature, and the specific surface area of DW-Ni-700 was much higher than that of DW-Ni-800. Therefore, DW-Ni-700 was chosen as the qualified candidate for the synthesis of GC.

3.2. Effect of Reaction Condition on the Synthesis of GC. Enough reaction time is required to obtain the maximum GC yield. However, the excessive reaction time can cause the beginning of the side reactions [37]. Thus, the effect of reaction time on the synthesis of GC was firstly investigated, and the result is shown in Figure 6(a). When the reaction time increased from $30 \mathrm{~min}$ to $60 \mathrm{~min}$, the glycerol conversion increased from $81.9 \%$ to $96.6 \%$, and the GC yield increased from $79.1 \%$ to $93.3 \%$. When the reaction time is longer than $60 \mathrm{~min}$, the GC yield and glycerol conversion changed slightly. Therefore, the suitable reaction time for the synthesis of GC by DW-Ni-700 was $60 \mathrm{~min}$.

High reaction temperature can provide enough reaction energy, whereas the excessive reaction temperature can lead to the decomposition or evaporation of reagents. The effect of reaction temperature on glycerol conversion and GC yield is shown in Figure 6(b). The conversion of glycerol and the

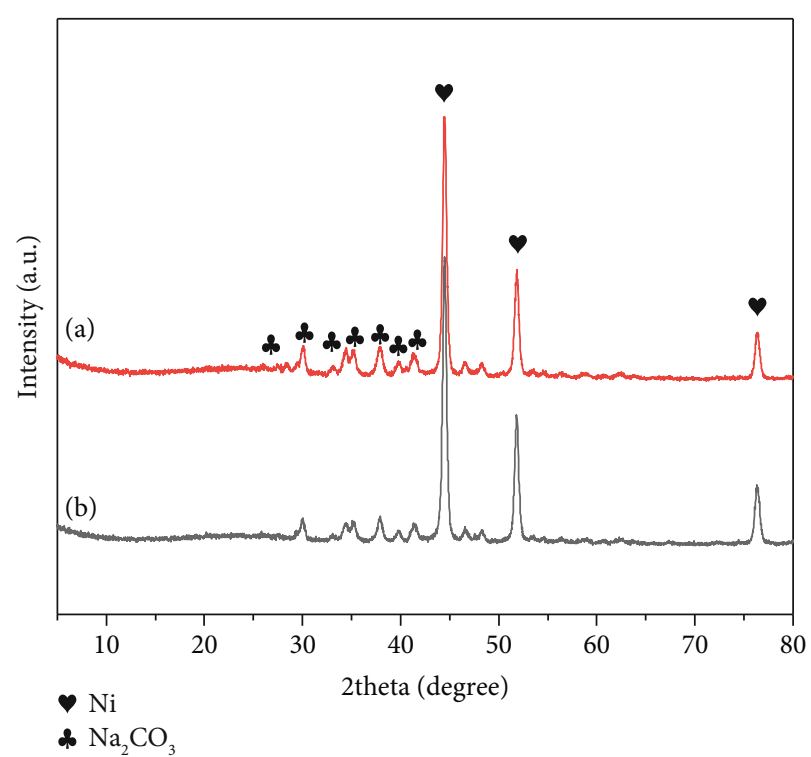

FIGURE 7: XRD patterns of the DW-Ni-700catalyst: (a) fresh DW$\mathrm{Ni}-700$ and (b) reused DW-Ni-700.

yield of GC increased gradually from $65^{\circ} \mathrm{C}$ to $80^{\circ} \mathrm{C}$. When the reaction temperature was $80^{\circ} \mathrm{C}$, the highest conversion of glycerol, $96.6 \%$, and GC yield, $93.2 \%$, was obtained. With the increase of temperature, the conversion of glycerol and the yield of GC decreased slightly. This was because of the fact that, when the reaction temperature was higher than $80^{\circ} \mathrm{C}$, the evaporation of DMC happened, leading to the fact that there was no enough DMC to react with glycerol $[7,38]$. Thus, $80^{\circ} \mathrm{C}$ was the suitable reaction temperature.

DMC to glycerol molar ratio can influence the diffusion of reagents to catalyst, which can consequently affect the conversion of glycerol and the yield of GC [39]. The effect of the molar ratio of DMC to glycerol on the reaction is shown in Figure 6(c). When the DMC to glycerol molar ratio increased from $1: 1$ to $3: 1$, the glycerol conversion and GC yield increased from $51.8 \%$ and $48.1 \%$ to $96.6 \%$ and $93.2 \%$, respectively. This result demonstrated that a relative high molar ratio of DMC to glycerol has a benefit for the formation of GC. When the molar ratio of DMC to glycerol was higher than $3: 1$, the GC yield hardly increased and the excessive DMC can decrease the utilization efficiency of feedstock. The DMC to glycerol molar ratio of 3:1 was most suitable for the reaction.

The effect of catalyst amount on the reaction is shown in Figure $6(\mathrm{~d})$. When the amount of catalyst, DW-Ni-700, increased from $1 \mathrm{wt} . \%$ to $5 \mathrm{wt} . \%$, the conversion of glycerol and the yield of GC increased from $84.3 \%$ and $81.1 \%$ to $96.6 \%$ and $93.2 \%$, respectively. When the catalyst amount was higher than $5 \%$, there was almost no change in the conversion of glycerol and the yield of GC. Catalyst can provide the necessary alkaline active sites for the synthesis of GC. However, when the catalyst amount was too high, it can lead to the agglomeration of catalyst and limit the further improvement of glycerol conversion and GC yield [40]. The suitable catalyst amount for the synthesis of GC was $5 \%$. 
TABLE 2: Comparison of the DW-Ni-700 with reported heterogeneous catalysts.

\begin{tabular}{lcccccc}
\hline Entry & Catalyst & Feedstock & Yield $_{f}(\%)^{\mathrm{a}}$ & Reuse times & $\mathrm{Yield}_{r}(\%)^{\mathrm{b}}$ & Ref. \\
\hline 1 & $\mathrm{LiCl} / \mathrm{CaO}$ & LiCl, CaO & 94.2 & 5 & 87.3 & {$[41]$} \\
2 & Ti-SBA-15 & SBA-15, titanium isopropoxide & 81.8 & 3 & 66.4 & {$[42]$} \\
3 & OPFA & Oil palm fuel ash & 94.5 & 5 & 81.5 & {$[3]$} \\
4 & $\mathrm{RM}-500$ & Red mud & 92.0 & 4 & 8.2 & {$[29]$} \\
5 & $\mathrm{CoFe}_{2} \mathrm{O}_{4} @(\mathrm{CaO}-\mathrm{ZnO})$ & $\mathrm{Co}\left(\mathrm{NO}_{3}\right)_{2}, \mathrm{Zn}\left(\mathrm{NO}_{3}\right)_{2}, \mathrm{CaCl}_{2}, \mathrm{Fe}\left(\mathrm{NO}_{3}\right)_{3}$ & 96.9 & 4 & 86.8 & {$[43]$} \\
6 & $\mathrm{DW}-\mathrm{Ni}-700$ & Diaper waste, $\mathrm{Ni}\left(\mathrm{NO}_{3}\right)_{2}$ & 93.2 & 4 & 89.6 & Present work \\
\hline
\end{tabular}

${ }^{\mathrm{a}} \mathrm{GC}$ yield when the fresh catalyst was applied. ${ }^{\mathrm{b}} \mathrm{GC}$ yield after the catalyst was recycled for several times.

3.3. Reusability of Catalyst. Reusability is an important property for heterogeneous catalyst. The good reusability of catalyst means the longer service life and the potential industrial application. Thus, the reusability of DW-Ni-700 was tested. In the reuse experiment, the catalyst was firstly used under the optimum reaction condition and then separated from the product by magnet. After being washed with methanol, the catalyst was dried in an oven at $100^{\circ} \mathrm{C}$ for 2 hours. The recovered catalyst was used in the next reaction cycle. The reuse experiment result of catalyst is shown in Figure S2. The fresh catalyst showed high glycerol conversion, $96.6 \%$, and GC yield, 93.2\%. After being used for four times, the glycerol conversion and GC yield decreased to $92.8 \%$ and $89.6 \%$, respectively. Both glycerol conversion and GC yield have decreased by less than $4 \%$. This result indicated the high reusability of DW-Ni-700.

In order to find out the reason for the decline of the catalytic performance of DW-Ni-700 after four times reuse, XRD analysis of fresh DW-Ni-700 and reused DW-Ni-700 was conducted. As shown in Figure 7, the diffraction peak of $\mathrm{Ni}$ did not change, while the diffraction peak of $\mathrm{Na}_{2} \mathrm{CO}_{3}$ changed slightly after the reuse experiment. The diffraction peak of $\mathrm{Na}_{2} \mathrm{CO}_{3}$ at $27.6^{\circ}$ can hardly be observed in the reused sample, indicating the leaching of $\mathrm{Na}_{2} \mathrm{CO}_{3}$ during the reaction. EDX is another technique which can investigate the leaching of the active site of catalyst [35]. It can be observed form Table S2 that the main surface elements of the fresh DW-Ni-700 were sodium, nickel, oxygen, and carbon, with weight percentage all higher than $12 \%$. The weight percentage of nitrogen, chlorine, and potassium was lower than 3\%. After the reuse experiment, the content of $\mathrm{Na}$ in DW-Ni-700 decreased by more than $7 \%$, indicating the leaching of $\mathrm{Na}_{2} \mathrm{CO}_{3}$ in the reaction. XRD and $\mathrm{EDX}$ investigation results indicated that the $\mathrm{Na}_{2} \mathrm{CO}_{3}$ leaching was the reason for the decrease of the catalytic ability of DW-Ni-700.

3.4. Comparison of DW-Ni-700 with Other Reported Heterogeneous Catalyst. Developing high-performance and low-cost heterogeneous catalyst is an important task in the field of GC synthesis. A lot of heterogeneous catalysts have been developed in the past decades. Table 2 shows the catalytic ability of some reported heterogeneous catalysts. The presented heterogeneous catalysts exhibited high catalytic ability with the GC yield higher than $90 \%$ when they were used in the GC synthesis. To prepare $\mathrm{LiCl} / \mathrm{CaO}$ and $\mathrm{Ti}$ -
SBA-15, the purchased chemical reagents were needed during the catalyst preparation process, which can increase the catalyst preparation cost. OPFA and RM-500 were prepared from waste materials. The negligible feedstock cost made them superior to $\mathrm{LiCl} / \mathrm{CaO}$ and Ti-SBA-15. However, OPFA and RM-500 can only be separated from the product by the filtration method, which can lead to the consumption of energy and the loss of catalyst. Moreover, the reusability of RM-500 was not satisfactory because GC yield decreased more than $70 \%$ after it was used for three times. When $\mathrm{CoFe}_{2} \mathrm{O}_{4} @(\mathrm{CaO}-\mathrm{ZnO})$ and DW-Ni-700 were used as the catalyst in the GC synthesis, magnetic separation can be carried out after the reaction, leading to the high separation performance compared with OPFA and RM-500. Considering the complex preparation process and relative high feedstock cost of $\mathrm{CoFe}_{2} \mathrm{O}_{4} @(\mathrm{CaO}-\mathrm{ZnO})$, the wide application of $\mathrm{CoFe}_{2}$ $\mathrm{O}_{4} @(\mathrm{CaO}-\mathrm{ZnO})$ was limited. Thus, the simple preparation process, high catalytic ability, and easy separation process made DW-Ni-700 a high-performance and low-cost MHC for the GC synthesis.

\section{Conclusion}

It is a simple method to prepare the MHC for the synthesis of GC from a polymeric waste, diaper waste, by impregnation and calcination techniques. The prepared catalyst, DW-Ni700 , was the composite of $\mathrm{Ni}, \mathrm{Na}_{2} \mathrm{CO}_{3}$, and carbon. The relative mild preparation condition, high total basicity $(4.7 \mathrm{mmol} / \mathrm{g})$, and specific surface area $\left(59.9 \mathrm{~m}^{2} / \mathrm{g}\right)$ of DWNi-700 made DW-Ni-700 an ideal catalyst for the synthesis of GC. When DW-Ni-700 was used in the synthesis of GC, the conversion of glycerol reached $96.6 \%$, and the yield of GC reached $93.2 \%$. Meanwhile, DW-Ni-700 can be magnetically separated from the product. After four times reuse of DW-Ni-700, the yield of GC decreased by less than $4 \%$. Compared with the reported heterogeneous catalyst, DWNi-700 is a low-cost and high-performance catalyst. The development of DW-Ni-700 provided a new way not only to prepare magnetic heterogeneous catalyst but also to utilize diaper waste.

\section{Data Availability}

The data (figures) that support the findings of this study are available upon request from the corresponding author. 


\section{Conflicts of Interest}

The authors declare that there is no conflict of interest regarding the publication of this paper.

\section{Acknowledgments}

Authors acknowledge the support from the Open Research Fund of the State Key Laboratory of Polymer Physics and Chemistry, Changchun Institute of Applied Chemistry, Chinese Academy of Sciences (2018-02), the General Project Fund of Liaoning Education Department (LJGD2019014), and the Key Laboratory for Catalyst Synthesis Technology of Polymer of Liaoning Province, China (2010-36).

\section{Supplementary Materials}

Figure S1: XRD of the catalysts prepared above $600^{\circ} \mathrm{C}$ in the $2 \theta$ range of $5^{\circ}-30^{\circ}$. Scheme $\mathrm{S} 1$ : the main possible reactions happened during the calcination process. Table S1: the detailed reaction condition. Figure S2: reusability of the DW-Ni-700 in the transesterification of glycerol with DMC. Table S2: elemental composition of the DW-Ni-700 and the reused DW-Ni-700. (Supplementary Materials)

\section{References}

[1] S. Wang, P. Hao, S. Li, A. Zhang, Y. Guan, and L. Zhang, "Synthesis of glycerol carbonate from glycerol and dimethyl carbonate catalyzed by calcined silicates," Applied Catalysis A, General, vol. 542, pp. 174-181, 2017.

[2] N. Özveren and M. Ö. Seydibeyoğlu, "The use of biodiesel residues for heat insulating biobased polyurethane foams," International Journal of Polymer Science, vol. 2017, Article ID 6310198, 14 pages, 2017.

[3] P. U. Okoye, S. Wang, W. A. Khanday, S. Li, T. Tang, and L. Zhang, "Box-Behnken optimization of glycerol transesterification reaction to glycerol carbonate over calcined oil palm fuel ash derived catalyst," Renewable Energy, vol. 146, pp. 2676-2687, 2020.

[4] C. Ardila-Suárez, D. Rojas-Avellaneda, and G. E. RamirezCaballero, "Effect of temperature and catalyst concentration on polyglycerol during synthesis," International Journal of Polymer Science, vol. 2015, Article ID 910249, 8 pages, 2015.

[5] L. Xu, S. Wang, P. U. Okoye et al., "Water glass derived catalyst for the synthesis of glycerol carbonate via the transesterification reaction between glycerol and dimethyl carbonate," Journal of the Serbian Chemical Society, vol. 84, no. 6, pp. 609-622, 2019.

[6] M. Aghbashlo, M. Tabatabaei, H. Rastegari, and H. S. Ghaziaskar, "Exergy-based sustainability analysis of acetins synthesis through continuous esterification of glycerol in acetic acid using Amberlyst ${ }^{\circledR 3}$ as catalyst," Journal of Cleaner Production, vol. 183, pp. 1265-1275, 2018.

[7] S. Wang, L. Xu, P. U. Okoye, S. Li, and C. Tian, "Microwaveassisted transesterification of glycerol with dimethyl carbonate over sodium silicate catalyst in the sealed reaction system," Energy Conversion and Management, vol. 164, pp. 543-551, 2018.

[8] X.-Y. Song, G. X. Pan, Y. W. Bai et al., "Preparation and electrochemical properties of biochar from pyrolysis of pomelo peel via different methods," Fullerenes, Nanotubes and Carbon Nanostructures, vol. 27, no. 5, pp. 453-458, 2019.

[9] B. Changmai, I. B. Laskar, and L. Rokhum, "Microwaveassisted synthesis of glycerol carbonate by the transesterification of glycerol with dimethyl carbonate using Musa acuminata peel ash catalyst," Journal of the Taiwan Institute of Chemical Engineers, vol. 102, pp. 276-282, 2020.

[10] X. Song, Y. Wu, F. Cai, D. Pan, and G. Xiao, "High-efficiency and low-cost $\mathrm{Li} / \mathrm{ZnO}$ catalysts for synthesis of glycerol carbonate from glycerol transesterification: The role of $\mathrm{Li}$ and $\mathrm{ZnO}$ interaction," Applied Catalysis A: General, vol. 532, pp. 7785, 2017.

[11] P. U. Okoye, A. Z. Abdullah, and B. H. Hameed, "Glycerol carbonate synthesis from glycerol and dimethyl carbonate using trisodium phosphate," Journal of the Taiwan Institute of Chemical Engineers, vol. 68, pp. 51-58, 2016.

[12] J. Granados-reyes, P. Salagre, and Y. Cesteros, "Effect of the preparation conditions on the catalytic activity of calcined $\mathrm{Ca} / \mathrm{Al}$-layered double hydroxides for the synthesis of glycerol carbonate," Applied Catalysis A, General, vol. 536, pp. 9-17, 2017.

[13] Y. Liu, S. Wang, H. Zhou et al., "Simultaneous removal of methyl orange and $\mathrm{Cr}$ (VI) using poiyethyleneiminemodified corncob-derived carbon meterial," BioResources, vol. 15, no. 4, pp. 7342-7356, 2020.

[14] Y. T. Algoufi and B. H. Hameed, "Synthesis of glycerol carbonate by transesterification of glycerol with dimethyl carbonate over K-zeolite derived from coal fly ash," Fuel Processing Technology, vol. 126, pp. 5-11, 2014.

[15] Y. Du, J. Gao, W. Kong et al., "Enzymatic synthesis of glycerol carbonate using a lipase immobilized on magnetic organosilica Nanoflowers as a catalyst," ACS Omega, vol. 3, no. 6, pp. 66426650, 2018.

[16] F. Zhang, X. H. Wu, M. Yao, Z. Fang, and Y. T. Wang, "Production of biodiesel and hydrogen from plant oil catalyzed by magnetic carbon-supported nickel and sodium silicate," Green Chemistry, vol. 18, no. 11, pp. 3302-3314, 2016.

[17] T. Su, J. Zeng, H. Gao et al., "One-pot synthesis of a chemically functional magnetic carbonaceous acid catalyst for fermentable sugars production from sugarcane bagasse," Fuel, vol. 262, p. 116512, 2020.

[18] K. Dhanalaxmi, R. Singuru, S. Mondal et al., "Magnetic nanohybrid decorated porous organic polymer: synergistic catalyst for high performance levulinic acid hydrogenation," ACS Sustainable Chemistry \& Engineering, vol. 5, no. 1, pp. 1033-1045, 2016.

[19] J. Mondal, T. Sen, and A. Bhaumik, " $\mathrm{Fe}_{3} \mathrm{O}_{4} @$ @mesoporous SBA-15: a robust and magnetically recoverable catalyst for one-pot synthesis of 3,4-dihydropyrimidin-2(1H)-ones via the Biginelli reaction," Dalton Transactions, vol. 41, no. 20, pp. 6173-6181, 2012.

[20] A. Rezayan and M. Taghizadeh, "Synthesis of magnetic mesoporous nanocrystalline $\mathrm{KOH} / \mathrm{ZSM}-5-\mathrm{Fe}_{3} \mathrm{O}_{4}$ for biodiesel production: process optimization and kinetics study," Process Safety and Environmental Protection, vol. 117, pp. 711-721, 2018.

[21] M. Tudorache, L. Protesescu, A. Negoi, and V. I. Parvulescu, "Recyclable biocatalytic composites of lipase-linked magnetic macro-/nano-particles for glycerol carbonate synthesis," Applied Catalysis A, General, vol. 437-438, pp. 90-95, 2012.

[22] U. Arena, F. Ardolino, and F. Di Gregorio, “Technological, environmental and social aspects of a recycling process of 
post-consumer absorbent hygiene products," Journal of Cleaner Production, vol. 127, pp. 289-301, 2016.

[23] Y. Budyk and A. Fullana, "Hydrothermal carbonization of disposable diapers," Journal of Environmental Chemical Engineering, vol. 7, no. 5, article 103341, 2019.

[24] H. Karimi and Q. L. Yu, "Valorization of waste baby diapers in concrete," Resources, Conservation and Recycling, vol. 153, p. 104548, 2020.

[25] R. Atchudan, T. N. J. I. Edison, S. Perumal, P. Thirukumaran, R. Vinodh, and Y. R. Lee, "Green synthesis of nitrogen-doped carbon nanograss for supercapacitors," Resources, Conservation and Recycling, vol. 102, pp. 475-486, 2019.

[26] S. Wang, L. Xu, L. Xu, C. Tian, and Y. Guan, "Optimization of process variables in the synthesis of tributyl citrate using a polyvinylpolypyrrolidone-supported brønsted acidic ionic liquid catalyst," International Journal of Polymer Science, vol. 2018, Article ID 1953563, 9 pages, 2018.

[27] K. Hu, H. Wang, Y. Liu, and C. Yang, " $\mathrm{KNO}_{3} / \mathrm{CaO}$ as costeffective heterogeneous catalyst for the synthesis of glycerol carbonate from glycerol and dimethyl carbonate," Journal of Industrial and Engineering Chemistry, vol. 28, pp. 334-343, 2015.

[28] S. Sandesh, G. V. Shanbhag, and A. B. Halgeri, "Transesterification of glycerol to glycerol carbonate using $\mathrm{KF} / \mathrm{Al}_{2} \mathrm{O}_{3}$ catalyst: the role of support and basicity," Catal. Lett., vol. 143, no. 11, pp. 1226-1234, 2013.

[29] B. Das and K. Mohanty, "A green and facile production of catalysts from waste red mud for the one-pot synthesis of glycerol carbonate from glycerol," Journal of Environmental Chemical Engineering, vol. 7, no. 1, article 102888, 2019.

[30] Y. Wan, Y. Lei, G. Lan, D. Liu, G. Li, and R. Bai, "Synthesis of glycerol carbonate from glycerol and dimethyl carbonate over DABCO embedded porous organic polymer as a bifunctional and robust catalyst," Applied Catalysis A: General, vol. 562, pp. 267-275, 2018.

[31] N. Jebakumar, I. Edison, R. Vinodh, K. H. Park, and Y. R. Lee, "An ultrasensitive photoelectrochemical biosensor for glucose based on bio-derived nitrogen-doped carbon sheets wrapped titanium dioxide nanoparticles," Biosensors and Bioelectronica, vol. 126, pp. 160-169, 2018.

[32] H.-L. Chang, Y. W. Bai, X. Y. Song et al., "Hydrothermal synthesis, structural elucidation and electrochemical properties of three nickel and cobalt based phosphonates as anode materials for lithium ion batteries," Electrochimica Acta, vol. 321, article 134647, 2019.

[33] Y. Yang, M. Wang, P. Shi, J. Wu, Y. Min, and Q. Xu, "Recycling of nitrogen-containing waste diapers for catalytic contaminant oxidation: occurrence of radical and non-radical pathways," Chemical Engineering Journal, vol. 384, 2020.

[34] K. Malins, "The potential of $\mathrm{K}_{3} \mathrm{PO}_{4}, \mathrm{~K}_{2} \mathrm{CO}_{3}, \mathrm{Na}_{3} \mathrm{PO}_{4}$ and $\mathrm{Na}_{2} \mathrm{CO}_{3}$ as reusable alkaline catalysts for practical application in biodiesel production," Fuel Processing Technology, vol. 179, pp. 302-312, 2018.

[35] S. Wang, J. Wang, P. Sun et al., "Disposable baby diapers waste derived catalyst for synthesizing glycerol carbonate by the transesterification of glycerol with dimethyl carbonate," Journal of Cleaner Production, vol. 211, pp. 330-341, 2019.

[36] B. Hazmi, U. Rashid, Y. H. Taufiq-Yap, M. L. Ibrahim, and I. A. Nehdi, "Supermagnetic nano-bifunctional catalyst from rice husk: Synthesis, characterization and application for con- version of used cooking oil to biodiesel," Catalysts, vol. 10, no. 2, p. 225, 2020.

[37] Y. T. Algoufi, U. G. Akpan, G. Kabir, M. Asif, and B. H. Hameed, "Upgrading of glycerol from biodiesel synthesis with dimethyl carbonate on reusable $\mathrm{Sr}-\mathrm{Al}$ mixed oxide catalysts," Energy Conversion and Management, vol. 138, pp. 183-189, 2017.

[38] W. K. Teng, G. C. Ngoh, R. Yusoff, and M. K. Aroua, "Microwave-assisted transesterification of industrial grade crude glycerol for the production of glycerol carbonate," Chemical Engineering Journal, vol. 284, pp. 469-477, 2016.

[39] W. A. Khanday, P. U. Okoye, and B. H. Hameed, "Biodiesel byproduct glycerol upgrading to glycerol carbonate over lithium-oil palm ash zeolite," Energy Conversion and Management, vol. 151, pp. 472-480, 2017.

[40] P. U. Okoye, S. Wang, L. Xu, S. Li, J. Wang, and L. Zhang, "Promotional effect of calcination temperature on structural evolution, basicity, and activity of oil palm empty fruit bunch derived catalyst for glycerol carbonate synthesis," Energy Conversion and Management, vol. 179, pp. 192-200, 2019.

[41] Y. Tang, Y. Y. Xue, Z. Li, T. Yan, R. Zhou, and Z. Zhang, "Heterogeneous synthesis of glycerol carbonate from glycerol and dimethyl carbonate catalyzed by $\mathrm{LiCl} / \mathrm{CaO}$," Journal of Saudi Chemical Society, vol. 23, no. 4, pp. 494-502, 2019.

[42] P. Devi, U. Das, and A. K. Dalai, "Production of glycerol carbonate using a novel Ti-SBA-15 catalyst," Chemical Engineering Journal, vol. 346, pp. 477-488, 2018.

[43] P. Zhang, Y. Chen, M. Zhu et al., "Acidic-basic bifunctional magnetic mesoporous $\mathrm{CoFe}_{2} \mathrm{O}_{4} @(\mathrm{CaO}-\mathrm{ZnO})$ for the synthesis of glycerol carbonate," Catalysis Letters, vol. 150, no. 10, pp. 2863-2872, 2020. 